\title{
¿Están desapareciendo del aire los aromas florales?*
}

$\mathrm{U}$ na flor bajo cualquier otro nombre ¿mantendría la dulzura de su olor? Tal vez no, si es que la contaminación atmosférica tiene algo que ver con el asunto. Investigadores de la Universidad de Virginia en Charlottesville informan que tres componentes comunes del esmog destruyen los aromas que despiden las flores para atraer a las abejas y a otros insectos polinizadores. De hecho, los aromas de las flores llegaban a una distancia hasta cuatro veces mayor en la década de los 1840 -cuando los científicos europeos comenzaron a documentar la contaminación por ozono- que bajo las condiciones actuales del aire, según los modelos de simulación operados por los investigadores. Dado que los insectos polinizadores dependen en parte de los aromas para encontrar las flores, la pérdida de las estelas aromáticas podría dificultar aun más a los insectos la localización de las fuentes de polen, lo cual haría peligrar tanto a los polinizadores

\footnotetext{
* Publicado originalmente en Environmental Health Perspectives, Volumen 116, Número 8, agosto 2008, página A334.
}

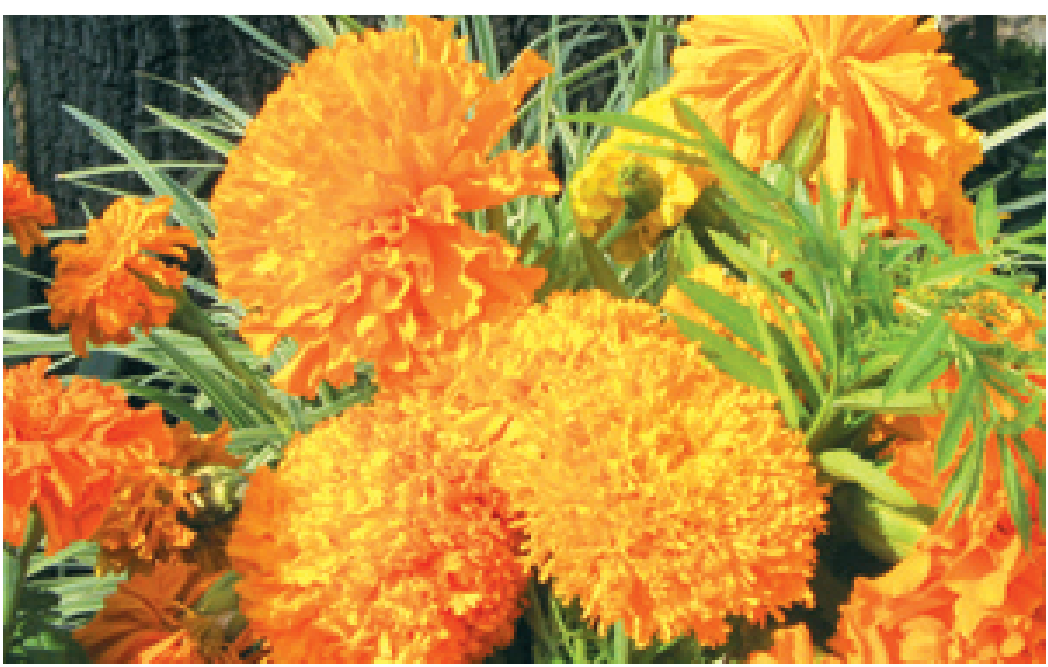

como a las cosechas. Los investigadores están iniciando pruebas de campo para ver si los aromas florales que el aire transporta en el mundo real corresponden con los resultados predichos por el modelo.

La vitalidad de las plantas depende de la polinización, según el primer investigador José Fuentes, un meteorólogo. Además, si se obliga a los polinizadores a pasar más tiempo buscando alimento y, no obstante, recolectan menos polen para alimentar a sus crías, las colonias de insectos pueden verse afectadas en el aspecto nutricional. Ambos problemas podrían tener impacto en nuestra provisión de alimentos. "Necesitamos preservar a los polinizadores porque proporcionan servicios útiles", dice Fuentes.

Ya se había establecido que cuando las moléculas aromáticas que van flotando en la dirección del viento se topan con contaminantes atmosféricos, las reacciones químicas que se suscitan alteran los aromas florales y contribuyen a la producción de compuestos como la acetona, el formaldehido y el monóxido de carbono. En el estudio en curso, Fuentes y sus colegas Quinn McFrederick y James Kathilankal consideraron el destino de tres hidrocarburos volátiles comunes emitidos por las flores cuando se encontraban con niveles crecientes de ozono, radicales hidroxilo y radicales nitrato. En el verano de 2002, los investigadores midieron la temperatura, la turbulencia de los vientos y otros factores que determinan el índice de emisión y el movimiento de los aromas florales en una granja experimental en Virginia. Observaron cómo estos factores afectaban la liberación del aroma de un campo de flores conocidas como "boca de dragón" que habían crecido de manera silvestre en la granja.

Los investigadores integraron estos datos a un modelo para probar diferentes escenarios de contaminación atmosférica, desde las condiciones que prevalecían en la década de los 1840 hasta las condiciones actuales durante el verano en las grandes ciudades del este de Estados Unidos, donde los niveles de ozono pueden exceder las $120 \mathrm{ppb}$ por volumen. En las condiciones de los años 1840, únicamente un $20 \%$ de los aromas se veían alterados por las reacciones químicas dentro de un radio de 1000 metros desde la fuente floral del aroma, en la dirección del viento. Sin embargo, incluso los incrementos ligeros de los contaminantes -comparables a la calidad del aire hoy en día en las áreas rurales que tienen pocas emisiones industriales-alteraron más del $40 \%$ de los aromas dentro de un radio de 500 metros desde la fuente floral. En el panorama más contaminado, únicamente un $25 \%$ de los 
aromas sobrevivieron en un radio de 300 metros en la dirección del viento. Fuentes y sus colegas reportaron estos hallazgos en la revista Atmospheric Environment, vol. 42, No 10 (marzo de 2008).

Los compuestos que se generan cuando los aromas florales se ven alterados químicamente ¿empeoran la contaminación atmosférica? "No hemos pensado aún en esto en [función de] la calidad del aire", dice Fuentes. Agrega que los hallazgos también suscitan inquietudes especiales en relación con el destino de los polinizadores nocturnos, tales como las palomillas, que dependen en gran medida del aroma para encontrar las flores (a diferencia de las abejas, que se valen tanto del color como del aroma durante el día).

Fuentes advierte que no deben aplicarse los nuevos hallazgos al trastorno del colapso de las colonias (CCD, por sus siglas en inglés), un fenómeno inexplicado que ha diezmado a las colonias de abejas en los últimos dos años. Dice que algunas de las mejores evidencias hasta la fecha sugieren que el CCD está más directamente relacionado con los agentes infecciosos y los plaguicidas. Sin embargo, señala, cualquier efecto de la contaminación atmosférica "probablemente constituye un factor adicional de estrés al que las abejas tienen que hacer frente."

Otros comportamientos de insectos que se orientan mediante los aromas químicos, como el apareamiento de los escarabajos, también podrían verse trastornados por la contaminación atmosférica. Por otra parte, algunas plantas pueden beneficiarse del deterioro de las señales transportadas por el aire: "Si los insectos no pueden oler las plantas, no pueden venir a comérselas", dice Jay Evans, un entomólogo investigador del Ministerio de Agricultura de Es- tados Unidos, quien llama al estudio de Fuentes "una hermosa mezcla de buena ecología y química".

Los polinizadores no son la única especie que lidia con los efectos olfativos de la contaminación atmosférica. En el número de Chemical Senses de enero de 2006, Robyn Hudson y sus colegas reportaron que los residentes de la Ciudad de México, una de las más contaminadas del mundo, tenían una capacidad considerablemente menor de detectar y distinguir entre los olores de los alimentos que los residentes de Tlaxcala, un área geográficamente similar con mucha menos contaminación atmosférica. Se observó esta diferencia en múltiples grupos de edades, aun cuando se eliminó del análisis a los fumadores, lo cual es muy sugestivo de un vínculo con los contaminantes que pueden dañar el epitelio olfativo.

Carol Potera

\section{Señal intensa para los efectos de los teléfonos celulares*}

$\mathrm{D}$ ado que hay 3 mil millones de usuarios de teléfonos celulares en todo el mundo, y más de $260 \mathrm{mi}-$ llones únicamente en Estados Unidos -entre ellos $46 \%$ de los niños estadounidenses de 8 a 12 años de edad, según las cifras de la empresa Nielsen Mobile publicadas el 10 de septiembre de 2008-, la exposición humana a la radiación de baja energía dentro del rango de 800 a 2000 megahertz está todo el tiempo a su máximo. El intento más reciente de estudiar de manera sistemática la evidencia epi- demiológica del riesgo incrementado de tumores cerebrales relacionado con el uso de teléfonos celulares indica que las repercusiones de este experimento global están saliendo a la luz. En un meta-análisis publicado en el número de mayo de 2008 del International Journal of Oncology, investigadores suecos encontraron asociaciones importantes entre el uso prolongado del teléfono celular y el riesgo de tumores cerebrales.

"Encontramos que el uso de los teléfonos celulares está vinculado a los gliomas [tumores cerebrales malignos] y a los neuromas acústicos [tumores benignos del nervio auditivo del cerebro], y éstos se están manifestando después de tan sólo diez años", dice el autor principal Lennart Hardell, oncólogo y epidemiólogo del cáncer del Hospital de la Universidad de Örebro, Suecia. Específicamente, en los estudios que incluyeron un mínimo de 10 años de exposición, se duplicó el riesgo de gliomas para las exposiciones ipsilaterales (del mismo lado) pero no para las contralaterales

\footnotetext{
* Publicado originalmente en Environmental Health Perspectives, Volumen 116, Número 10, octubre 2008, página A422.
} 